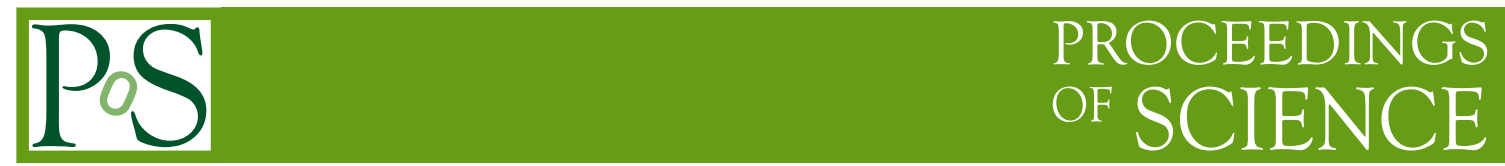

\title{
Front-end and Back-end in radioastronomy
}

\author{
Enzo Natale* \\ INAF Istituto di Radioastronomia \\ E-mail: nataleearcetri.astro.it
}

\section{Renzo Nesti}

Osservatorio Astrofisico di Arcetri

E-mail: nestiearcetri.astro.it

In this paper we describe high performance microwave components commonly used in large bandwidth receivers for the centimetric and millimetric frequency range. Limitations to the ideal receiver sensitivity due to different sources of noise are discussed. Finally two different focal plane arrays, which offer a major way to increase the data rate of astronomical observation of extended sources, are presented.

First MCCT-SKADS Training School

September 23-29, 2007

Medicina, Bologna Italy

${ }^{*}$ Speaker. 


\section{Introduction}

Purpose of these notes are to give some information on the technical aspects to be faced in designing and building heterodyne receivers for radioastronomy. Of course not all aspects would be covered in detail in the short time we have; certainly not in the few pages of this paper, so we will focus our attention on arguments which seems to us of relevant importance. One must also consider the fact that the sensitivity of modern radioreceivers is approaching, at least in some bands, the quantum limit, so the only way to increase the time productivity of large antennas is to increase the "instantaneous" field of view or, in other words, the number of detectors. This can be achieved with the deployment of focal plane arrays or phased arrays. Both these approaches will require strong efforts in solving problems involved both in building complex receivers and in the handling, storing and analyzing huge amount of data. Here we deal with single dish systems approach because of their flexibility and because they can be also used as elements of synthesis arrays like VLBI and similar networks.

\section{The basic receiver}

The system noise temperature of a receiver at the focus of an antenna is mainly determined, in absence of strong celestial sources (Sun, Moon, planets, ...), by the cosmic background radiation modified by the atmosphere, the atmospheric emission and the receiver noise. In frequency bands free of strong atmospheric absorption lines mainly due to the Oxygen and Water vapor, the antenna temperature when looking at the sky is about one order of magnitude below or even less that the ambient temperature $(300 \mathrm{~K})$. Consequently the receiver noise temperature is typically responsible of the system noise temperature; its reduction by cryogenic cooling offers a very effective mean to increase the receiver sensitivity. Fig. 1 shows schematically a single pixel dual polarization cryogenic receiver; basically it consist of a feed horn to collect the radiation coming from the antenna, some passive microwave components, two Low Noise Amplifiers (LNA) and finally an IF processor to down convert the radiofrequency into an intermediate frequency for further processing. The passive microwave architecture following the feed horn typically comprise a directional coupler to inject a signal used to calibrate the receiver and an Orthomode Transducer (OMT) to separate the linear polarization components of the sky signal: sometimes circular polarization components are required and in this case a Differential Phase Shifter (DPS) is needed in front of the OMT to form together what is usually called a polarizer. To achieve low noise performances the front-end is generally cooled down to about $10-20 \mathrm{~K}$ by means of mechanical refrigerators. The required thermal insulation between the front-end and both the IF processor and Noise source, is usually guaranteed by using stainless steel waveguides or cables. At the same time infrared filters are usually needed between the horn and the vacuum window to block most of the thermal power coming from the ambient. These filters must provide high transparency at the operating radiofrequency and high opacity in the infrared band. Summarizing, to constrain the thermal load on the cryogenerator to an acceptable level, in order to achieve predetermined working temperatures, one has to use a thermal shield, typically fastened to the intermediate temperature stage of the cryogenerator (about 70K), and to minimize the 


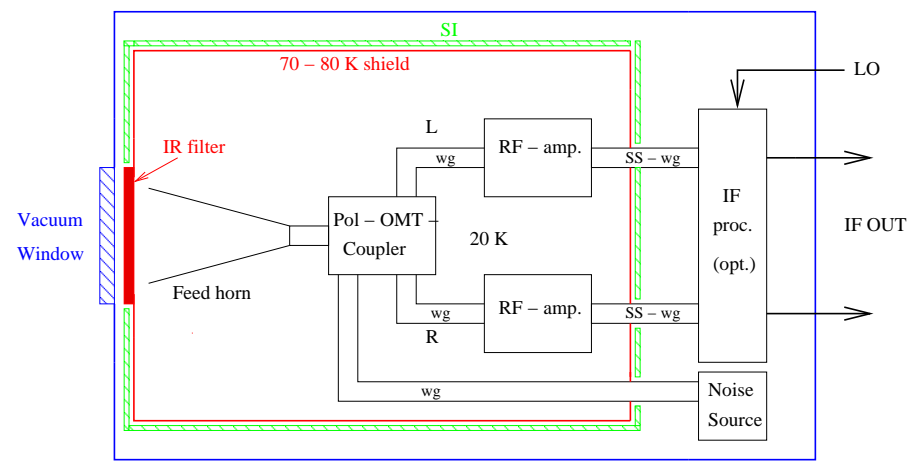

Figure 1: Layout of a single beam, dual polarization, cryogenic receiver

- radiative power coming through the vacuum window by using suitable infrared blocking filters;

- thermal exchanges between the dewar walls and the shield by using superinsulation [3];

- power due to the Joule effect and the thermal conductivity of the harness.

In the following a brief description of the basic components are given.

\subsection{Feed horn}

A feed horn, like any other antenna, may be seen as an energy format transducer between the electromagnetic radiation and the waveguide propagation. Among the large number of feed horn types (see for instance in [9] for an almost complete review) we briefly discuss the conical corrugated one, sketched in Fig. 2a, which is the most used at the secondary foci of large antennas for its unique features. As a matter of fact this kind of horn typically shows highly symmetric beam pattern, very low cross polarization (less than about $-35 \mathrm{~dB}$ ) and high return loss $(\geq 30 \mathrm{~dB})$ over a quite broad (about 30\%) bandwidth. The first part of the corrugated region, the mode launching section (see Fig. 2a), principally determines the input matching (or return loss) and the proper excitation of the fundamental hybrid mode $\mathrm{HE}_{11}$ propagating in the corrugated section from the $\mathrm{TE}_{11}$ of the exciting circular waveguide. The physical dimensions (the horn length and aperture diameter) and the overall profile of the flare section determine the radiation pattern and hence the antenna illumination, characterized by the edge taper, that is the relative field level at the edge, compared to the on axis maximum at the center of the reflector. The corrugation geometry, in particular the groove depth and its period, mainly affects the cross-polarization performances. The exact analysis of the propagation in a corrugated horn is analytically quite complex [5] and highly sophisticated software [12] is required for good accuracy. As an example of attainable performances, the measurements obtained on the feed horns designed for the SRT (Sardinia Radio Telescope) 18 - 26 $\mathrm{GHz}$ multibeam, developed in the framework of the Faraday project ${ }^{1}$, are reported [6]:

\footnotetext{
${ }^{1}$ FARADAY project was funded by European Union in the Framework Programme 5.
} 

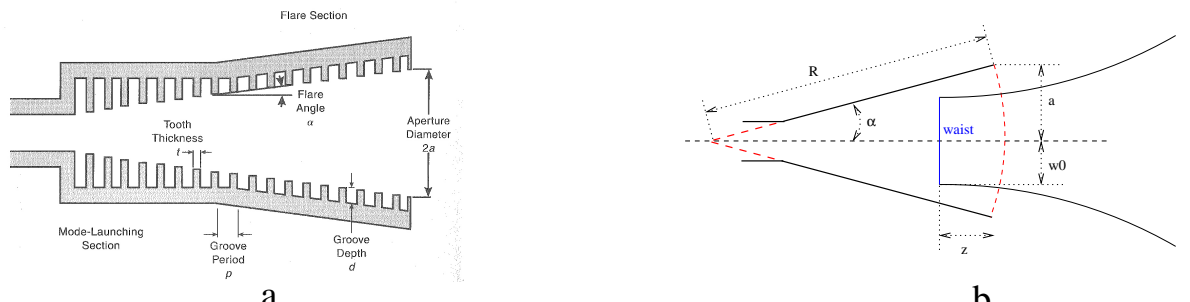

$\mathrm{b}$

Figure 2: a) Cross section of a corrugated feed horn. b) Geometrical and Gaussian fundamental beam mode parameters

$\begin{array}{ll}\text { Return loss } & : \geq 30 \mathrm{~dB} \\ \text { Insertion loss } & : \leq-0.1 \mathrm{~dB} \\ \text { Off axis cross polarization } & : \leq-30 \mathrm{~dB} \\ \text { Bandwidth } & : 30 \% \text { or larger }\end{array}$

and illustrated in Fig. 3

To evaluate the performances achievable at the focus of a large antenna, i.e. an antenna whose diameter $D$ is much larger than the wavelength $\lambda$, it is sufficient to give here some results based on the approximation of the electromagnetic field distribution in terms of Gaussian beam modes (in [9] there is a very good and complete treatment of the argument).

In this approximation, it can be shown [9] that, for a not too large flare angle $\alpha$ and an aperture radius $a$ greater than the wavelength (see Fig. $2 \mathrm{~b}$ ) a feed horn produces a gaussian beam whose waist radius is $w=0.644 a$ located inside the horn at a distance $z$ approximately equal to $1 / 3$ of the horn length. In these conditions about $98 \%$ of the power radiated by the horn can be associated with the fundamental Gaussian beam mode. Using the standard formulae for Gaussian mode propagation [9], it becomes simple to compute the antenna illumination (the taper $T$ ) and consequently the Full Width to Half Maximum beam width in the sky (FWHM) which, for a in-focus system and unblocked aperture, is given by:

$$
\triangle \theta_{F W H M}=(1.02+0.0135 T(d B)) \frac{\lambda}{D} \mathrm{rad}
$$

\subsection{DPS and OMT}

To increase the capability and versatility of the antenna system, dual polarization operation is often required. This can be done using a single feed (operating in dual polarization mode) with the separation of the two orthogonal linearly polarized signals by using OMTs. Further, if it is desired to separate the circular polarizations, a wide-band Differential Phase Shifter (DPS), which transforms circular to linear polarizations, can be inserted between the horn and the OMT. The performances generally required for astronomical observations to the combination DPS+OMT are: 


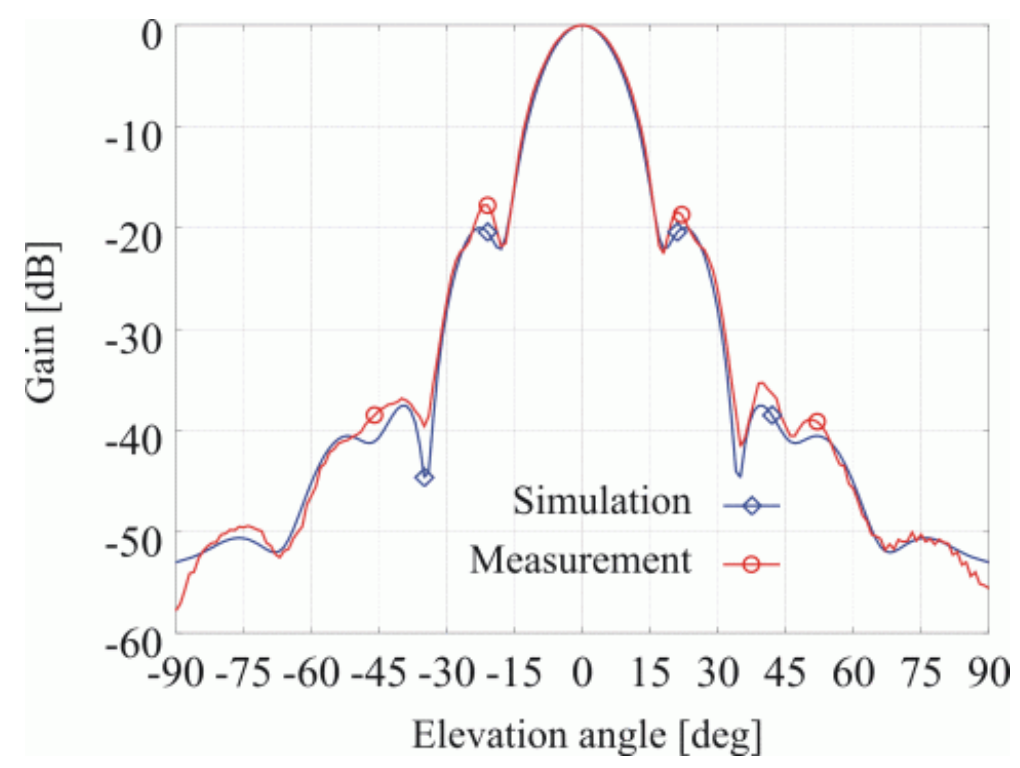

Figure 3: Copolar E-plane pattern of the feed horn for the 18-26 GHz multibeam
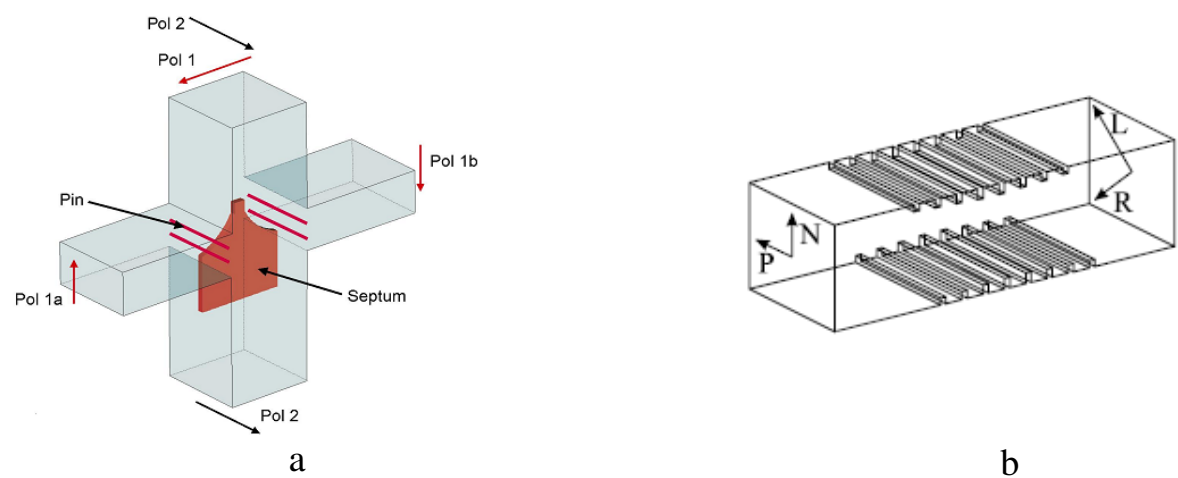

Figure 4: a) Bøifot dual junction and b) Differential Phase shifter.

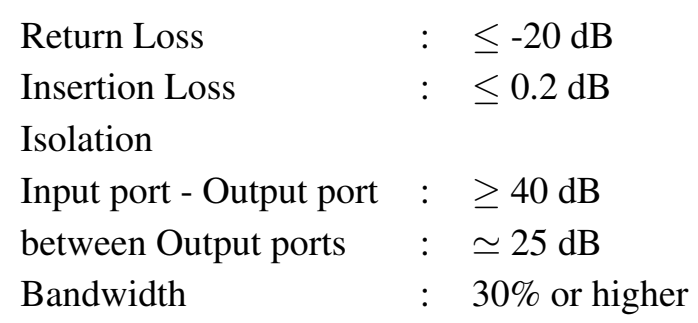

There are various OMT designs (for a review see for instance [8]) commonly used operating with acceptable performance over a limited bandwidth, typically between $10 \%$ and $20 \%$. Much wider bandwidths, of the order of $30 \%$ or higher, to match the bandwidth attainable by circular corrugated horns, can be obtained by using, for instance, the rectangular waveguide dual junctions as developed by Bøifot [2] (depicted in Fig. 4a) or its derivation, see for instance [1] and the references therein cited, or the turnstile solution [14]. 


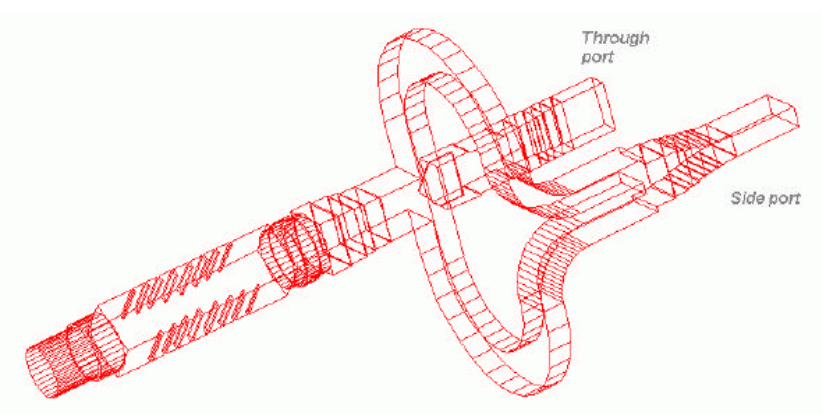

Figure 5: Complete EM model of the polarizer including DPS, OMT and waveguide interfaces

The Bøifot design requires a thin septum and two matching pins to optimize the performances. More recently $[13,15]$ a modified version has been successfully designed to simplify its realization by eliminating the matching pins and by optimizing the shape and thickness of the septum. When observing in circular polarization mode a DPS must be inserted between the feed horn and the OMT. The DPS will provide the transformation, by inserting a 90deg phase lag, between the P and $\mathrm{N}$ linear polarization (see Fig. 4b) so that if the Left Hand (LHCP) or the Right Hand Circular Polarization impinges on the P-N port (on the left side) then at the other port (on the right side) we have, respectively, the $\mathrm{L}$ or the $\mathrm{R}$ linear polarization, $45 \mathrm{deg}$ rotated with respect to the $\mathrm{P}$ and $\mathrm{N}$ one. Fig. $4 \mathrm{~b}$ shows, among the various possibilities, the chosen solution [15] that uses a square waveguide with corrugation on only two facing sides producing the desired differential phase shifting between the two polarization P and N. An EM model of the complete polarizer (DPS + OMT), designed and manufactured for the $18-26 \mathrm{GHz}$ band is shown in Fig. 5 and the ambient temperature overall passive front-end performances are reported in Fig. 6. It must be stressed that the Insertion Loss information has to be read as the envelop of curve b) giving a value around $-0.4 \mathrm{~dB}$ and that we expect a much lower value at cryogenic temperature due to the increase of the electrical conductivity of the metals (see. for instance [25]).

\subsection{Low Noise RF amplifiers}

The next critical component of a high performance receiver is the Low Noise Radio Frequency Amplifier. Typical performances required to a cryogenic LNA are: 

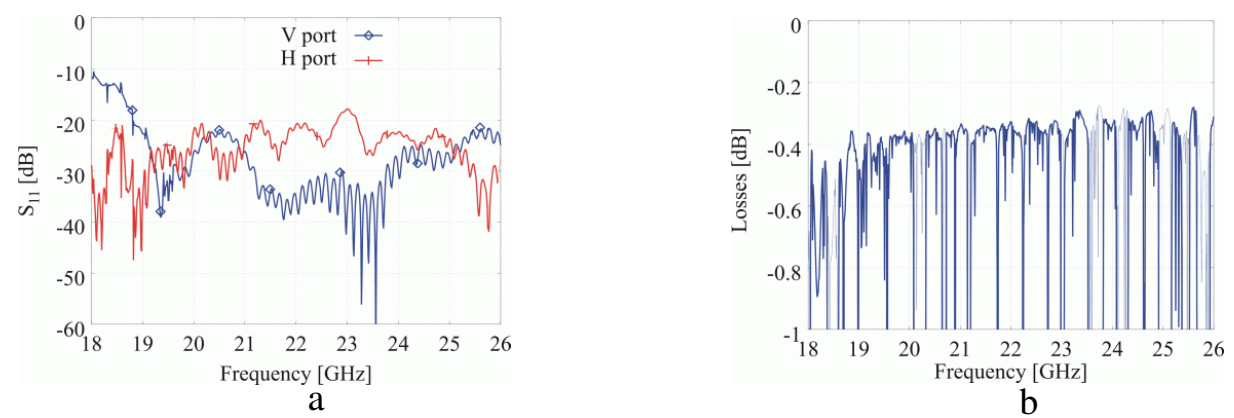

Figure 6: Performances of the overall system made by feed horn, DPS and OMT a) Return Loss and b) Insertion losses. True insertion loss is the upper envelope of the curve, negative spikes are measurement artifacts.

$\begin{array}{ll}\text { Gain } & : \geq 30 \mathrm{~dB} \\ \text { Gain flatness } & : \leq 1-2 \mathrm{~dB} \\ \text { Input return loss } & : \leq-15 \mathrm{~dB} \\ \text { Bandwidth } & : 30 \% \text { or larger } \\ \text { Power Out @ 1dB Compression } & :+5 \mathrm{dBm} \\ \text { Working temperature } & : \simeq 20 \mathrm{~K}\end{array}$

In these last years a big jump has been done in the construction of LNA due to the possibility to design and realize amplifiers with the integrated circuit technology and the availability of the Indium Phosphide technology. For a review of the state of art in the field see, for instance, [19, 20].

\subsection{Intermediate Frequency Processor}

Receivers for radioastronomy generally are of superheterodyne type and as consequence requires an intermediate frequency processor mainly for two purposes:

- accurate definition of the receiving band

- conversion of the radio frequency band to an intermediate frequency (IF) (normally equal for all receivers) for easy interfacing to the back-end.

Figure 7 gives the block diagram of a typical IF processor. The signal band centered at $v_{s k y}$ coming from the LNA is mixed with a signal of frequency $v_{L O-1}$ coming from a local oscillator (LO-1). At the mixer output there are present two bands respectively centered at $v_{u p}=v_{s k y}+v_{L O-1}$ and $v_{\text {down }}=\left|v_{\text {sky }}-v_{L O-1}\right|$. Taking into account the up-conversion, we have at mixer output two sky bands whose center frequencies are distant $2 \times v_{L O-1}$. If $v_{L O-1}$ is high enough than it is relatively simple to filter out only one sky frequency band as can be simply verified using the number marked in the figure; a second mixer provides the final conversion compatible with the back-end.

\section{Receiver sensitivity}

The observation of an astronomical source generally requires long integrations since the signals are deeply embedded in the noise. To extract the weak signals, synchronous detection (signal 


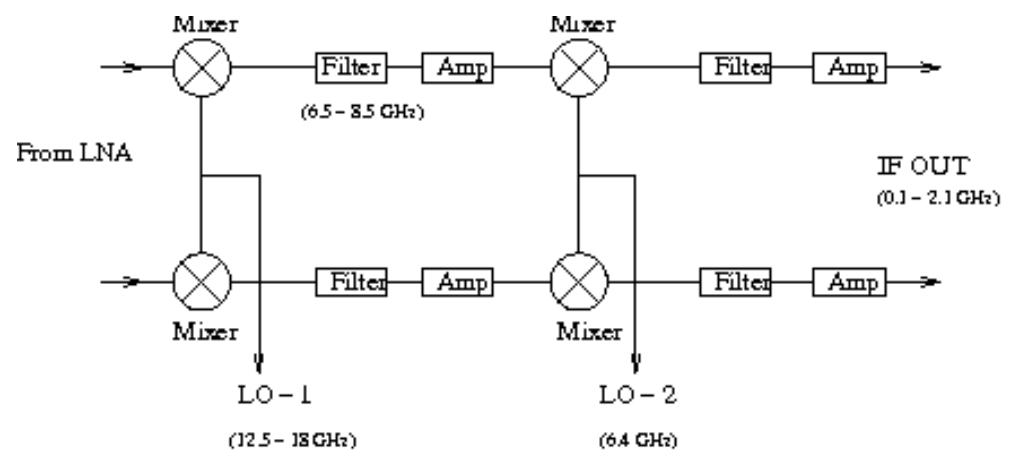

Figure 7: IF processor structure. In parenthesis the appropriate frequencies for the $18-26 \mathrm{GHz}$ receiver are marked

on - signal off) is typically employed to circumvent instabilities in the receiving system, including the atmosphere. For extended sources this is typically done by slewing the entire telescope back and forth, whereas in the case of point sources within the field of view of the telescope, nutating the secondary mirror is often employed. If the noise in the receiving system is completely uncorrelated (white), it turns out that the chopping frequency (modulation) has no effect on the signal to noise ratio. But in practice the low frequency noise power spectrum of a real detecting system (atmosphere + antenna + receiver) is no longer white because of the presence of $1 / f^{\alpha}$ noise, with $\alpha$ ranging form about 0.6 up to about 2, originating from the electronics and from the low frequency noise or drifts (correlated noise).

Fig. 8 shows a block diagram of a basic receiver where the system noise temperature $T_{s y s}$ is defined as the sum of all the noise sources due to the $2.7 \mathrm{~K}$ cosmic background as modified by the atmosphere $\left(T_{b k}\right)$, the atmospheric emission $\left(T_{a t m}\right)$, the contribution due to the far sidelobes of the beam $\left(T_{\text {opt }}\right)$ and the receiver noise temperature $\left(T_{\text {rec }}\right)$ :

$$
T_{s y s}=T_{b k}+T_{a t m}+T_{o p t}+T_{r e c}
$$

It can be shown $[11,21]$ that, for an ideal receiver, i.e. when only the white noise is present, the relation between the system noise $T_{s y s}$ and the rms fluctuation $\Delta T$ at the output of the receiver can be written as:

$$
\frac{\Delta T}{T_{s y s}}=\frac{1}{\sqrt{B \tau}}
$$

where $B$ is the bandwidth of the radio frequency filter and $\tau$ is the low-pass filter time constant. The noise bandwidth $B_{v}$ of some typical filters can be found in [11].

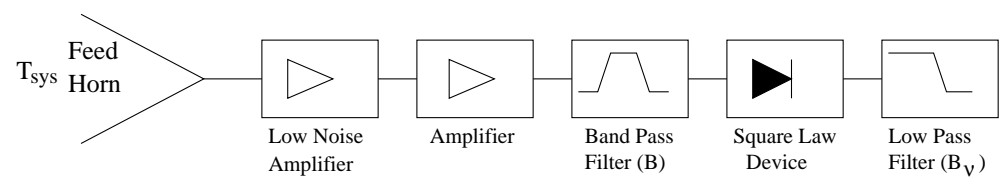

Figure 8: Principal components of a microwave receiver 
If we take into account the contributions induced by other noise sources like the LNA gain fluctuation $\Delta G$ and the fluctuations of $T_{\text {sys }}$ itself, due, for instance to atmospheric instabilities, then the above relation can be generalized as [26]:

$$
\frac{\Delta T}{T_{s y s}}=\left(\frac{1}{B \tau}+\left(\frac{\Delta G}{G}\right)^{2}+\left(\frac{\Delta T_{\text {sys }}}{T_{\text {sys }}}\right)^{2}\right)^{0.5}
$$

To fully characterize the complete receiver system (receiver+antenna+atmosphere) one must know the power spectra of all noise sources or, alternatively, the value of the variance of $\Delta T / T_{s y s}$ versus the integration time $\mathbf{T}$. In real observations, the wanted source signal $X(\mathbf{T})$, with $\mathbf{T}$ the integration time, is given by

$$
X(\mathbf{T})=\frac{1}{\mathbf{T}} \sum_{j=0}^{n}\left(x_{s}(j)-x_{r}(j)\right)
$$

with $n$ the number of samples of $x_{s}(j)$ and $x_{r}(j)$ respectively the measurements of the sky region with the source in the field of view and of a reference blank sky region (no source).

It can be shown [22] that the variance of $X(\mathbf{T})$ can be written as:

$$
\sigma^{2}(\mathbf{T})=C_{1} \mathbf{T}+C_{2}+C_{3} / \mathbf{T}
$$

where $C_{1}, C_{2}$ and $C_{3}$ are the coefficients for the drift noise, $1 / f$ noise and white noise.

In practice the last term in the above equation dominates for short integration times and the variance decreases as $1 / \mathbf{T}$, as expected for white noise. For longer integration times, the drift will dominate as shown by the term $C_{1} \mathbf{T}$. In that case, the variance starts to increase with a slope which is experimentally found to be between 1 and 2 . On certain occasions, it is observed that the variance plateaus at some constant level, denoted by the constant $C_{2}$. This is representative of $1 / f$ noise. Plotting $\sigma^{2}(\mathbf{T})$ versus $\mathbf{T}$ on a log-log plot, referred to as an Allan plot, demonstrates the usefulness of this approach in analyzing the noise statistics. The minimum in the plot gives the so called Allan time, the crossover from white noise to $1 / f$ and/or drift noise. Note that the Allan time is a function of the RF bandwidth $B$; as matter of fact $C_{3} \simeq 1 / B$.

As an example in Fig. 9 is reported the Allan plot computed from nine data sets recorded from the central receiver of the 18-26 multibeam. In this plot one can clearly identify three different regions: one with a $1 / \mathbf{T}$ slope, a central region where is clearly visible a plateau $(1 / f$ noise $)$ and then an increase of the variance $\left(\approx 1 / f^{2}\right.$ or drift noise $)$; the "knee" frequency $f_{k}=1 / \mathbf{T}$, equal about to 3 - $10 \mathrm{~Hz}$, can be recognized. This result imply that the low frequency noise power spectra of the receiver itself will require an ON-OFF modulation frequency well above about $3-10 \mathrm{~Hz}$ to meet the sensitivity as derived by the radiometric noise only.

The way to reduce the impact of the $1 / f^{\alpha}$ noise strongly depends on the type of observation and on the origin of the noise, i.e. from turbulent variation in emission from atmospheric water vapour (see for instance [4]) or from gain fluctuation of the LNA.

In the low frequency region and for spectroscopic observations, where $B$ is of the order $1-50$ $\mathrm{KHz}$, the predominant noise is the radiometric one. This imply that slow position switching can be tolerated. This case will not be considered anymore. 


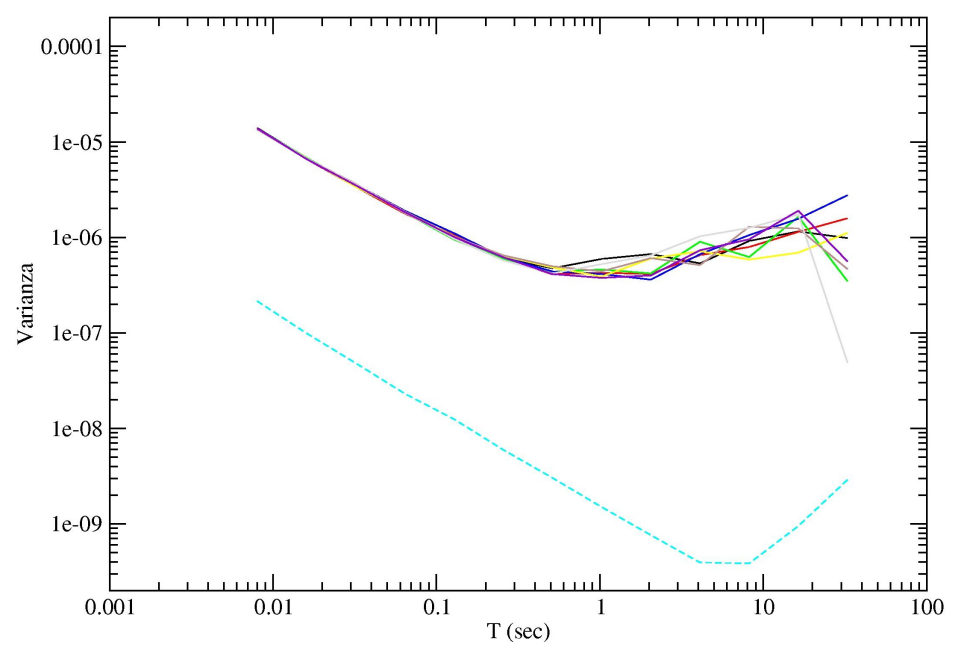

Figure 9: Allan plot of nine data set relative to the central receiver of the $18-26 \mathrm{GHz}$ multibeam. The dotted line is relative to the measuring system only.

For large bandwidth $(B \simeq 0.1-2 G H z)$ continuum observations there exist a number of modulation techniques to subtract the atmospheric emission depending also on the size of the source; i.e. if the source is smaller or much larger than the antenna beamwidth. In the case of point-like source the techniques usually adopted are:

a) the beam switching by nutating the subreflector in the "ON" position (source in the beam) and in the "OFF" position (reference position) and

b) the position switch in which the entire telescope is moved between "ON" and "OFF".

If the source is larger than at least few beams, then it is possible to use the On-The-Fly technique which is a quick scanning of the source allowing subtraction of the atmospheric emission if the positions at both ends of the scan are free of astronomical emission. For a good low frequency noise rejection it is strongly required that the modulation frequency (ON-OFF or the Nyquist sampling time in OTF) must be higher than $f_{k} \simeq 1 /$ Allan time ; a requirement that could be hard to achieve with position switch or even with large subreflector beam switching. The relative efficiencies of these methods are deeply analyzed by Schieder \& Kramer [22] using the Allan variance method.

When the LNA gain fluctuation is larger than the atmospheric noise, it is possible to reject to some extent the low frequency noise at expense of the final signal to noise ratio. Among the various techniques available for the gain fluctuation mitigation one can mention, for instance, the noise adding receiver and the single beam Dicke and correlation receivers [11]. It is worth noting that these techniques have no effects on all the noise sources external to the receiver itself like, for instance, the atmospheric fluctuations. In the following, as an example, a brief description is given of the noise adding radiometer sketched in Fig. 10.

The switch $\mathrm{S}$, which inject the noise in the receiver, is driven at a frequency $f$ higher than $f_{k}$. As a result we have at the receiver output a periodic signal at the modulation frequency $f$ whose levels are:

$$
s_{1}=k G T_{s y s} \quad \mathrm{~S} \text { open }
$$




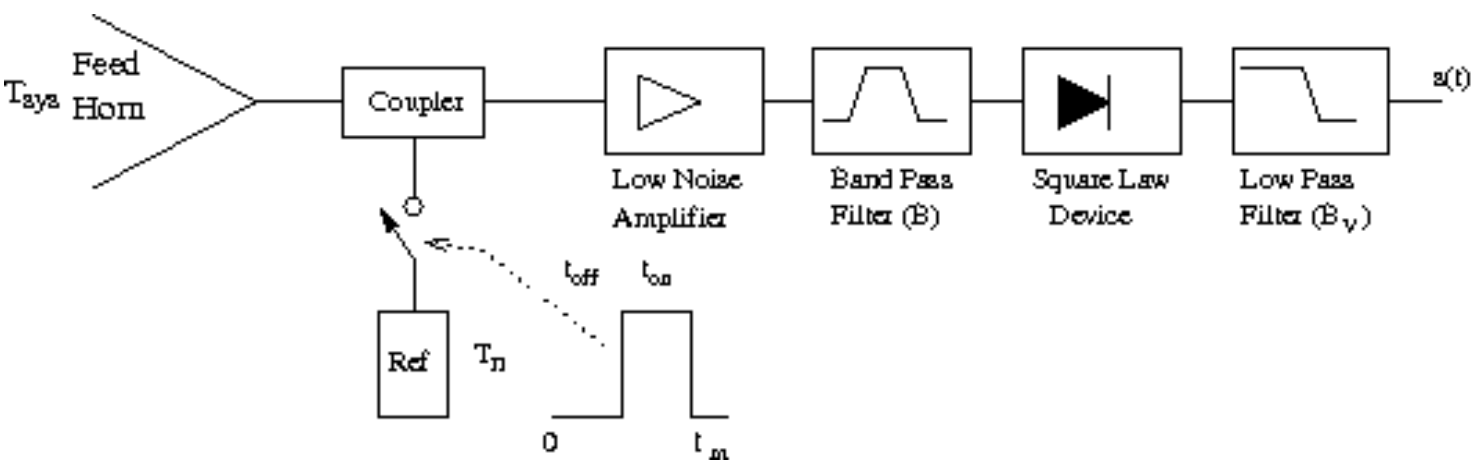

Figure 10: Block diagram of the noise adding radiometer.

$$
s_{2}=k G\left(T_{s y s}+T_{n}\right) \quad \text { S closed }
$$

with $k$ a proportionality factor and $G$ the receiver gain (fluctuating with the time).

With simple algebra on obtain:

$$
\begin{aligned}
T_{s y s} & =\frac{s 1}{s_{2}-s_{1}} T_{n} \\
\left(\frac{\Delta T_{s y s}}{T_{s y s}}\right)^{2} & =\frac{1}{B \tau}\left(1+\frac{s_{2}^{2}+s_{1}^{2}}{\left(s_{2}-s_{1}\right)^{2}}\right)
\end{aligned}
$$

Further, if we put $T_{n}=x T_{s y s}$, we can rewrite eq. 2 as

$$
\frac{\Delta T_{s y s}}{T_{s y s}}=\sqrt{\frac{2}{B \tau}}\left(1+\frac{1}{x}+\frac{1}{x^{2}}\right)^{0.5}
$$

i.e. the injected noise temperature $T_{n}$ should be of the same order of the temperature to be measured to have a not too high decrease in signal to noise ratio. As matter of fact, assuming $x=1$, the decrease of signal to noise ratio is 2.5 .

\section{How many receivers?}

The rate at which astronomical information can be extracted from large dishes when observing spatially extended sources is mainly limited by the number of independent beam on the sky and, consequentely, by the number of receivers. State of art receivers are approaching the fundamental limit sensitivity and the real observing sensitivity is limited both by the $1 / f$ receiver noise and by the atmospheric instabilities. In the case of extended sources, maps of the source brightness distribution are made by moving the single beam in a regular fashion over the source. The rate at which data is acquired can be substantially increased by having multiple detectors in the focal plane of the telescope, each with a distinct beam on the sky. The potential gain in instrument productivity that can be realized by the deployment of multiple-beam receiver greatly exceed that which may come through other technological development in the near future.

In the following we briefly discuss both "dense" packed array made by many small antenna elements (smaller than half wavelength) and "single horn" array made by a number of different receivers. 


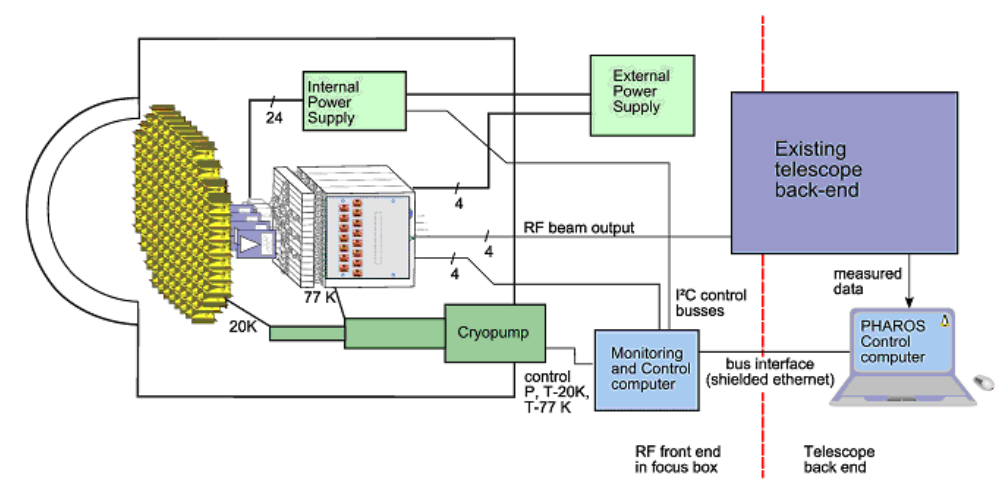

Figure 11: PHAROS overall system [23]. The large field of view together with the physical dimension of the array prevented the use of flat window; instead a Plexiglas $16 \mathrm{~mm}$ thick emispherical radome of $340 \mathrm{~mm}$ in diameter has been used. The RF transmission of the window, including the radome and infrared filtering, will be better than $95 \%$ in the $5-7 \mathrm{GHz}$ frequency range.

\subsection{Dense array}

FPA dense arrays are distinguished from traditional single-horn feed arrays by the fact that the antenna consists of an array of small (of the order of half wavelength) antenna elements. Multiple beams are formed by electronically summing the signals from different groups of elements. The beam properties can be optimized over a wide range of frequencies by electronically controlling element phases and amplitudes leading to high aperture efficiencies and low spillover losses of the radio telescope. The flexibility of the FPA also enables correction of surface errors of the dish and RFI (Radio Frequency Interference) mitigation. The multi beam capabilities of the FPA enable an increased field of view leading to a higher survey speed of the telescope.

Full sampling focal plane arrays have been demonstrated at $1.4 \mathrm{GHz}$ by Fisher et al. [7] and at 6 $\mathrm{GHz}$ by Ivashina et al. [10]. An example of a cryogenic dense array for the $5-7 \mathrm{GHz}$ band is PHAROS $^{2}[18,23,24]$ in which the antenna array and the beam former are cryogenically cooled for low noise operation. An overall view of the system is shown in Fig. 11.

The antenna array is designed to operate at prime focus of radiotelescopes with focal ratio $F=\mathbf{f} / D$ ranging from 0.3 to 0.5 , where $\mathbf{f}$ and $D$ are respectively the focal length and the diameter of the radiotelescope. The total number of antenna elements are 364 of which the 24 central elements are active; the number of simultaneous available beams will be 4 . The antenna pitch $18.6 \mathrm{~mm}$ (approximately $\lambda / 2$ at $8.0 \mathrm{GHz}$ ). The antenna structure is shown in Fig. 12; in the left side of the figure the location of the elements, both active and inactive, are shown, while in the right side a 3D view is provided. The number of inactive (dummy) elements around the active part of the array (see Fig. 12a ) is a significant factor because it determines the amount of edge effects that will influence system performance, especially for the outer beams. It also affects the outer dimensions of the antenna array. The "dummy" elements have no consequence to the beam former complexity because they will be terminated with $50 \mathrm{Ohm}$ resistors, which will exhibit only a small noise contribution due to the operating temperature of $20 \mathrm{~K}$.

\footnotetext{
${ }^{2}$ PHAROS (PHased Arrays for Reflector Observing Systems) project was funded by European Union in the Framework Programme 6 .
} 

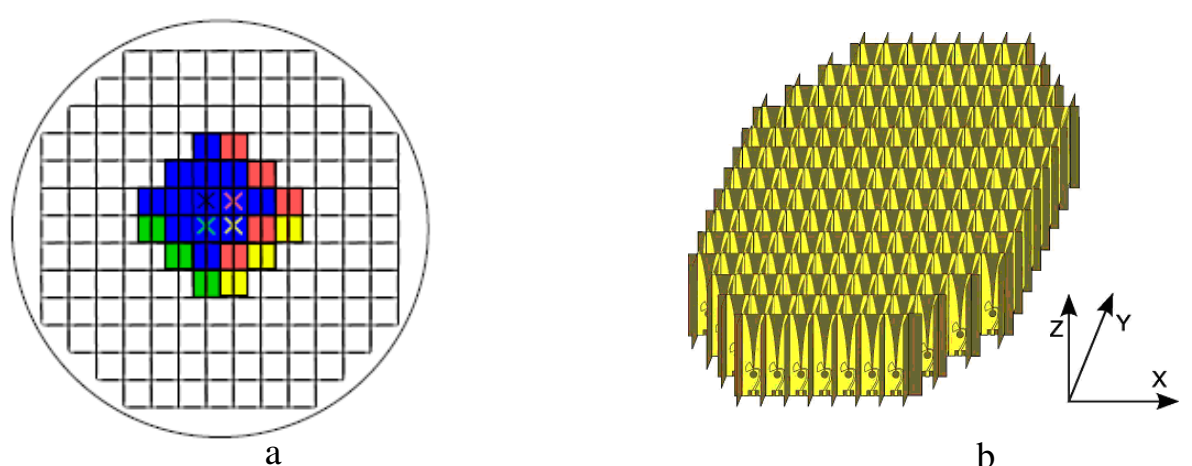

$\mathrm{b}$

Figure 12: PHAROS dense array using Vivaldi elements. a) Front view of the antenna with the 24 active elements indicated in different colors to form 4 beams and dummy elements (in white). The edge elements are removed for fitting in the $320 \mathrm{~mm}$ circular dewar window [24]. b) The complete PHAROS FPA antenna will be built up of 14 Printed Circuit Boards (PCB) in X direction and 14 PCBs in Y direction. Central PCBs will contain 13 Vivaldi elements.

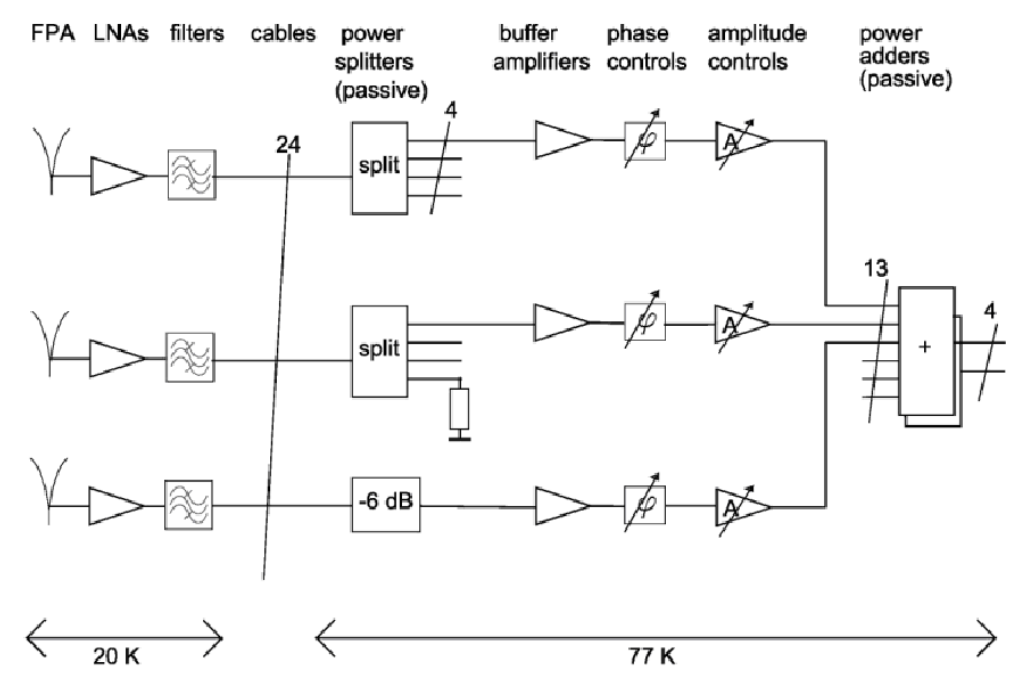

Figure 13: PHAROS beamformer block diagram [23]

RF beam forming is implemented for four beams each being a linear combination of 13 (see Fig. 12 a) Vivaldi elements each with an appropriate amplitude and phase. The beam forming circuits are built using highly symmetrical structures. A schematic overview can be found in Figure 13. The beam-former structure consists of several stages, including: a) band-pass filter to reduce possible out-of-band signal, b) power splitter, c) phase shifter and amplitude modulator for each element of each beam signal and d) power combiners for combining element signals into beams.

The four output signals are connected to the telescope existing back-end.

\subsection{Single horn array}

Current technology capabilities still prevent the use of the above described dense array at higher frequencies. The only possibility is to build-up array by assembling together a certain number of single channel (dual polarization) receivers. There is a quite large number of these 


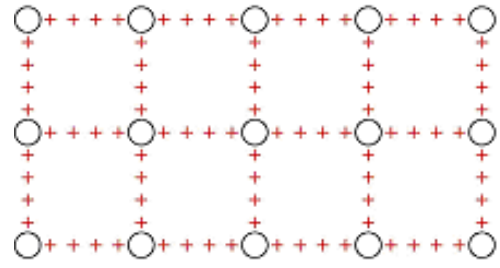

a

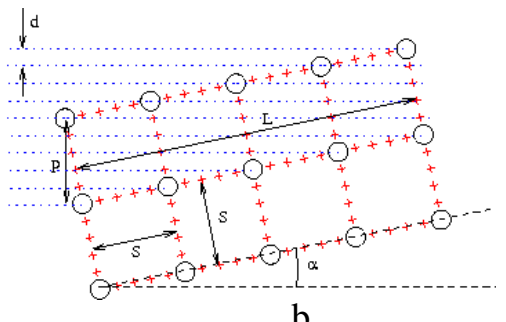

$\mathrm{b}$

Figure 14: a) Rectangular feed array arrangement. The crosses indicates the positions to full sample the sky; the circles the actual positions of the beams. $b)$ Tilted array $(3 \times 5$ pixels $)$

kind of array from about $20 \mathrm{GHz}$ to very high frequencies. For the sake of simplicity we briefly describe the structure of an hypothetical multibeam for the $36-50 \mathrm{GHz}$ band.

In the following we assume the use of conventional corrugated feed horns as receiving elements arranged in a square array at the secondary focus of the Medicina antenna whose main dish diameter is $D=32 m$ and focal ratio $F=\mathbf{f} / D=3.04$ with $\mathbf{f}=97 m$ the Cassegrain equivalent focal length.

It can be shown [9] that, to obtain a good coupling efficiency, the input radius $\mathrm{R}$ of the feed horn should be approximately $R=F \lambda=21.35 \mathrm{~mm}$ with $\lambda$ the operation wavelength of $7 \mathrm{~mm}(43 \mathrm{GHz})$. For such a horn, using Gaussian beam approximation, one can show [9] that the taper at telescope will be $9 \mathrm{~dB}$ with a corresponding efficiency of about 0.8 and a FWHM of $1.142 \lambda / D \simeq 45$ arcsec. To have a full sampling in the sky, the distance between the beams in the sky should be about equal to the Nyquist rate of $0.5 \lambda / D$. This limit translates in the focal plane to a distance $d=0.5 \lambda F$, hence, in the optimum efficiency conditions, we have a minimum under sampling given by the ratio $(2 \lambda F) /(0.5 \lambda F)=4$. In practice, due to mechanical constraints imposed by the horn construction and physical dimensions of the passive front-end components (Directional Coupler, DPS, OMT, ...) the horn inter-distance is larger than $2 \lambda F$ with a consequent under sampling factor larger than 4 . One can safely assume that it is possible to install the horns with an axis inter-distance $S=2 R+10 \mathrm{~mm} \simeq 53 \mathrm{~mm}=2.5 F \lambda$ so that the angular distance on the sky between two adjacent horn is $\theta=S / \mathbf{f}=113 \operatorname{arcsec}$. The actual under sampling, due to horn inter-distance, is then 5. Fig. 14a shows this condition for square arrangement where the horn positions are marked by circles and the beams positions for Nyquist sampling rate are marked by crosses. To full sample the sky we need 25 telescope positions. An alternative possibility to full sample the sky is the use of the On The Fly (OTF) technique with a rotation of the array with respect the scanning direction. Fig. 14b shows, as an example, a square array with an under sampling factor of 5; to fully sample the sky it is possible to choose a minimum number $n=5$ of elements in the scanning direction and a tilting angle $\alpha$ satisfying the condition of uniform sky sampling at Nyquist rate. It can be shown that [16] $\alpha$ should obey the relation $\alpha=\tan ^{-1}(1 / n)$, with $n$ the feed elements in the row. Using this arrangement together with the OTF technique, there is no advantage in an increase of the number of columns of the array, while the number of rows can be increased taking into account only the antenna corrected field of view. Assuming a $5 \times 5$ array, we have an instantaneous field of view of 0.13 squared-degrees and an occupation in the focal plane of about 300 squared-wavelengths which imply a window diameter of about $430 \mathrm{~mm}$.

Similar considerations can be drawn for hexagonal arrays. 


\section{Conclusions}

To increase the observing efficiency one has to to recover a significant fraction of the information available in the focal plane of large radio telescopes; as a consequence, large arrays, of the order of several tens of receivers, are needed in the millimetric bands. The near future efforts must, hence, be directed towards the making of high performance complete receivers far less expensive and time consuming to construct and test.

ACKNOWLEDGMENTS The authors gratefully acknowlwdges many stimulating discussions with A. Orfei and G. Comoretto.

\section{References}

[1] R. Banham ,G. Valsecchi, L. Lucci, G. Pelosi, S. Selleri, V. Natale, R. Nesti and G. Tofani, G., Electroformed Front-end at $100 \mathrm{GHz}$ for Radioastronomical Applications, Microwave Journal 48, n.8, 112, August 2005

[2] A.M. Bøifot, E. Lier and T. Shaug-Petersen, Simple and Broadband Orthomode Transducer, Proc. IEE, part H, Vol.137, n. 6, pp. 396-400, Dec. 1990.

[3] R.R. Conte, Elements de Cryogenie, Masson\&C C Editeurs, Paris, 1970

[4] S. Church, M.N.R.A.S., 272, 551, 1995

[5] P.J.B. Clarricoats and A.D. Olver, Corrugated Horn for Microwave Antennas, Peter Peregrinus, London, 1984

[6] Faraday - IRA Final Report, 2007

[7] J.R. Fisher and R.F. Bradley, R.F., Full-Sampling Focal Plane Arrays in Imaging at Radio through Submillimeter Wavelengths, ASP Conference Series, Vol. 217, J.G. Mangum and S.J.E. Radford Editors, 2000

[8] G.G. Gentili, R. Nesti, G. Pelosi and S. Selleri, Orthomode Transducers in The Wiley Encyclopedia of RF and Microwave Engineering, John Wiley \& Sons, (New York, NY, USA), Vol. 4, 2005

[9] P.F. Goldsmith, Quasioptical Systems: Gaussian Beam Quasioptical Propagation and Applications, IEEE Press, Piscataway, 1998

[10] M.V. Ivashina, J. Simons, and J.G. Bij de Vaate, Efficiency Analysis of Focal Plane Arrays in Deep Dishes, Experimental Astronomy 17, pp.149-162, 2004.

[11] J.D. Kraus, Radio Astronomy, McGraw-Hill Book Company, 1966

[12] L. Lucci, R. Nesti, G. Pelosi, and S. Selleri, Corrugated Horns in The Wiley Enciclopedia of RF and Microwave Engineering, John Wiley \& Sons, (New York, NY, USA), Vol. 4, 2005

[13] G. Narayanan, and N.R. Erickson, Design and performance of a novel full-waveguide band orthomode transducer, $13^{\text {th }}$ Symposium On Space THz Technology, Cambridge,MA, 2002

[14] A. Navarrini and R.L. Plambeck, A Turnstile Junction Waveguide Orthomode Transducer, IEEE MTT 54, n. 1, Jan 2006

[15] Nesti, R. et. al. The 22 GHz Polarizer Technical Report FARFI1 02/04, 2004 
[16] A. Orfei, et al. Feasibility study for a multifeed in the 43GHz band, IRA Internal Report n. 401, 2007

[17] R. Padman, Optical Fundamentals for Array Feeds in Multi-Feed Systems for Radio Telescopes ASP Conference Series, Volume 75, D.T. Emerson and J.M. Payne Editors, 1995

[18] PHAROS project (http://www.pharos-eu.org)

[19] M.W. Pospieszalski, Extremely Low-Noise Amplification with Cryogenic FETs and HFETs: 1970-2004, NRAO ELECTRONICS DIVISION Internal Report NO. 314, 2005

[20] M.W. Pospieszalski, and E.J. Wollack, Characteristics of broadband InP HFET millimeter wave amplifiers and their applications in radioastronomy receivers, in Proc. of ESA Workshop on mm-wave, Helsinki, May 1998

[21] Rohlfs, K., Wilson, T.L., Tools of Radio Astronomy, Springer, 1996

[22] R. Schieder, and C. Kramer, Optimization of heterodyne observations, A\&A 373, 746, 2001

[23] J. Simons, J.G. Bij de Vaate, M.V. Ivashina, M. Zuliani, V. Natale, and N. Roddis, Design of a focal plane array system at cryogenic temperatures, The European Conference on Antennas and Propagation: EuCAP 2006, ESA SP-626, Nice, 6 - 10 November 2006

[24] J. Simons, PHAROS System specifications Ver.0.7, June 2006

[25] G.K. White, Experimental Techniques in Low-Temperature Physics, Clarendon Press, Oxford, 1979

[26] E.J. Wollack, High-electron-mobility-transistor gain stability and its design implications for wide band millimeter wave receivers, Rev. Sci. Instrum. 66, August 1995, 4305 